\title{
Unobtrusive Non-Contact Detection of Arrhythmias using a "Smart" Bed
}

\author{
Ch. Brüser
}

\begin{abstract}
We present an instrumented bed for unobtrusive, non-contact monitoring of cardiac and respiratory activity. The system presented here is based on the principle of ballistocardiography (BCG), and measures cardiopulmonary vibrations of the body by means of an electromechanical foil (EMFi) attached to the mattress. Using our system, a clinical study with 13 participants was conducted to assess the BCG's ability to distinguish atrial fibrillations from normal sinus rhythms. By computing a time-frequency representation of the recorded signals based on parametric autoregressive estimators, we can show clear qualitative differences between normal and arrhythmic BCG episodes. The same distinctive features could also be observed when applying our method to a simultaneously recorded reference ECG. Our results suggest that ECG and BCG both contain the same basic information with respect to the presence of atrial fibrillations, and that a bed-mounted BCG sensor can indeed be used to detect atrial fibrillations.
\end{abstract}

Keywords: ballistocardiography, arrhythmia, bed, unobtrusive home-monitoring, atrial fibrillation.

\section{Introduction}

Cardiovascular diseases in general and heart failure in particular are among the commonest reasons for hospitalization in the industrialized countries [1]. In order to deal with the growing number of patients, there is a need for technical solutions which enable personalized monitoring and treatment, preferably at home. In recent years, the bed has emerged as a promising place for long-term monitoring of cardiopulmonary activity at home, as virtually everyone spends a significant portion of the day in bed. Instrumented beds could also be applied in the general wards of hospitals, where fully automatic, unobtrusive monitoring systems could reduce the workload of the staff and increase the safety of the patients.

A promising approach for measuring cardiopulmonary activity is the integration of highly sensitive mechanical sensors into the bed-frame or mattress which record the vibrations of the body caused by the mechanical activity of the heart and by the respiratory movements of the thorax.

This basic principle, known under the term "ballistocardiography" (BCG), was first reported in the late 19th century [2]. Through improvements in sensor technologies and digital signal processing techniques, the field has gained renewed interest in recent years. Using a variety of different sensors, including but not limited to strain gauges $[3,4], \mathrm{PVDF}$ and EMFi sensors [5,6], accelerometers [7], hydraulic [8] and pneumatic sensors $[9,10]$ as well as optical devices [11], BCG systems have been integrated into objects of daily life, such as beds [4-12], chairs [13] and even weighing scales [3]. These systems share the common advantage that they are unobtrusive and do not require direct skin contact, unlike for example, a conventional ECG. User errors, unlike, incorrectly attached electrodes can mostly be avoided by bed-based BCG systems, which do not require any user interaction or professional supervision to perform nightly measurements over extended periods of time. Hence BCG systems are very well suited for long-term monitoring.

While there have been significant contributions from various sensor modalities for BCG recording, as well as a few studies evaluating modern BCG systems, using healthy subjects, there is currently a lack of research on whether these systems actually provide clinically useful information when measuring real patients. After all, the answer to this question will decide whether or not these systems come into use in clinical practice. Arrhythmias, in particular, have so far been regarded as undesirable artefacts in many publications. We therefore devised a clinical study with the explicit goal of evaluating our BCG system's ability to detect cardiac arrhythmias. For our study we focused on atrial fibrillation (AF), since it is the most common type of arrhythmia [14]. During atrial fibrillation, the two upper chambers of the heart (atria) fibrillate and do not perform coordinated contractions [15]. AF is a marker for other severe illnesses such as congestive heart failure [16]. Long-term monitoring of AF episodes might enable an early detection of worsening conditions in heart failure patients. 
In the remainder of this paper, we first introduce our BCG measurement system (Section 2.1). Then we describe the design of the clinical study that was performed to acquire BCG data from real arrhythmia patients (Section 2.2). Next, we present the signal processing techniques that were applied to evaluate the acquired data (Section 2.3). We conclude by discussing the results and presenting our conclusions (Sections $3 \& 4$ ).

\section{Materials and Methods}

\subsection{BCG Acquisition System}

The BCG acquisition system used in this study consists of a single electromechanical-film (EMFi) sensor [17] $(30 \mathrm{~cm} \times 60 \mathrm{~cm}$, thickness $<1 \mathrm{~mm})$ and the acquisition electronics for amplifying and digitizing the analog sensor signal. Mechanical deformation of the electromechanical film generates a charge, $\Delta Q$, which is proportional to the dynamic force, $\Delta F$, acting along the thickness direction of the sensor

$$
\Delta Q=k \Delta F
$$

where $k$ is the sensitivity coefficient. The resulting charge is amplified by a charge amplifier, and is then digitized with 12 bits at a sampling frequency of $128 \mathrm{~Hz}$.

The EMFi foil is mounted on the underside of a thin foam overlay, which is then placed on top of the mattress of a regular bed. Due to its thinness and its flexible properties, the presence of the sensor is almost imperceptible to the person lying in the instrumented bed. However, owing to the sensitivity of the EMFi foil, cardiopulmonary movements of the person lying in bed can be recorded. In order to obtain optimal signals, the sensor is mounted in a fixed position under the thorax region (see Figure 1). A short segment of a signal recorded by our BCG system containing three heart beats is shown in Figure 2. Vertical dotted lines indicate the time points at which $\mathrm{R}$ peaks occurred in the reference ECG.

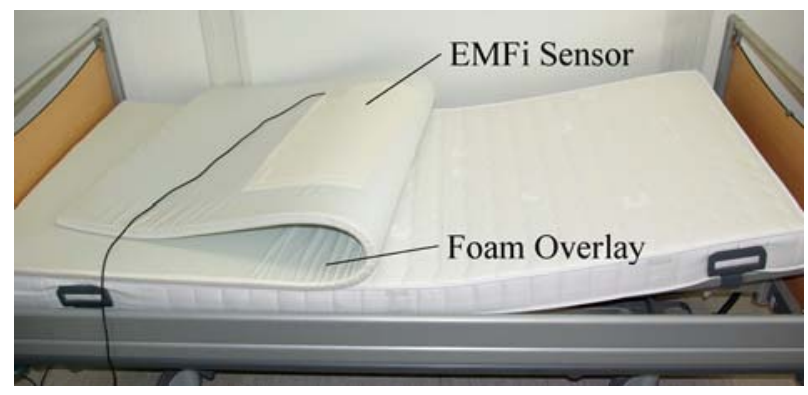

Fig. 1: Picture of the EMFi BCG sensor attached to the bottom of the thin foam overlay

\subsection{Measurement Scenario}

In order to assess whether atrial fibrillations and sinus rhythm can be distinguished in a BCG recording, the following study was performed at the University Hospital in Aachen, Germany. The study was approved by the ethics board of the University Hospital Aachen (ref. number: EK075/10, date: 05.05.2010). A total of 13 patients ( 3 female, 10 male, age: $63.6 \pm 16.3$ years, BMI: $\left.28.6 \pm 4.1 \frac{\mathrm{kg}}{\mathrm{m}^{2}}\right)$ who were visiting the hospital to undergo ambulatory treatment for atrial fibrillation gave their informed written consent and were included in our study. To return the patients' hearts to a regular sinus rhythm, a routine procedure called synchronized electrical cardioversion [18] was performed on each patient. During this procedure, an electrical current is administered to the heart. Unlike defibrillation, the initial current dose is smaller and the shock is triggered by the R-peak in the ECG in order to reduce the risk of induced ventricular fibrillation.

For the entire duration of their treatment, the subjects were placed in a hospital bed instrumented with an EMFi foil sensor, as described above. In addition to the BCG, a 3-lead reference ECG was recorded with a sampling rate of $500 \mathrm{~Hz}$. BCG and ECG data was continuously acquired before, during, and after the procedure. The mean length of the individual BCG recordings is 45 minutes.

This measurement scenario has the major advantage that it allows the BCG of the same patient to be recorded while exhibiting the pathology (i.e. arrhythmia/atrial fibrillations) as well as when the patient's heart is returned to a normal sinus rhythm. Hence an inter-personal as well as an intra-personal comparison of the BCG signal during arrhythmias and during normal rhythms is possible.

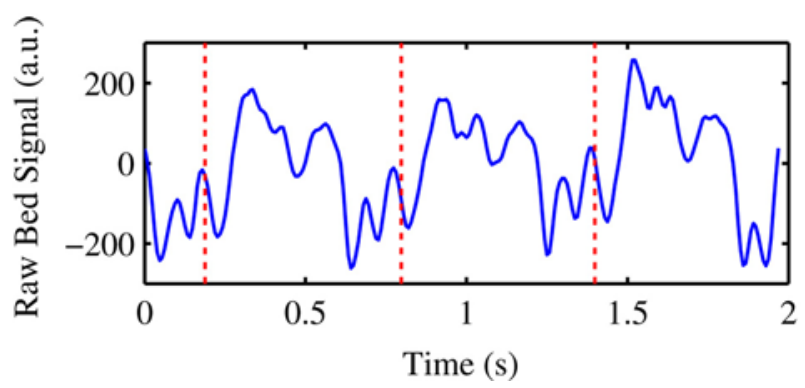

Fig. 2: Exemplary trace of three heart beats recorded by the bed sensor. Vertical lines indicate the occurrence of $\mathrm{R}$ peaks in the simultaneously recorded reference ECG

\subsection{Signal Analysis}

\section{PSD Estimation using AR Models}

Autoregressive (AR) models are a common choice for parametric estimation of the power spectral den- 
sity (PSD) of a signal [19]. First, a given signal, $x(n), n \in[0, N-1]$, is modelled as the output of a discrete, all-pole, infinite impulse response (IIR) filter whose input is white noise, $w(n)$, of variance $\sigma^{2}$ :

$$
x(n)=-\sum_{k=1}^{p} a_{k} x(n-k)+w(n) .
$$

Thus, for an $\operatorname{AR}(p)$ model of $p$-th order, only the filter coefficients $a_{1}, \ldots, a_{p}$ and the noise variance $\sigma^{2}$ need to be estimated to fully describe this process. After estimating these parameters, the PSD, $P(f)$, of the modelled process can be computed as:

$$
P(f)=\frac{\sigma^{2}}{\left|1+\sum_{k=1}^{p} a_{k} e^{-j 2 \pi f p}\right|^{2}}
$$

A number of different AR parameter estimation methods are known in the literature [19]. These are typically based on minimizing the estimate of the prediction error power. Popular estimators include the Yule-Walker method and Burg's method. For our analysis which follows, we have chosen to use the Burg estimator [20].

Autoregressive spectral estimation can provide better modelling of the peaks in the PSD than nonparametric methods, especially when dealing with short signal lengths [19]. This improvement comes at the cost of a less accurate description of the valleys of the PSD. When dealing with quasi-periodic signals measuring cardiac activity, however, this is a valid trade-off, as we are primarily interested in the peaks of the PSD. However, this advantage of AR spectral estimators exists only when the assumption of an underlying AR process is indeed valid for the given signal. Furthermore, the model order needs to be carefully chosen to achieve high quality estimates.

\section{Spectrogram}

Since biosignals, such as ECG and BCG, are also highly non-stationary in nature (especially in the presence of arrhythmias), a deeper insight into the properties of these signals can be achieved by analysing their time-frequency distributions. A common approach to obtain a time-varying spectral representation of a signal is to divide the signal into smaller (overlapping) epochs and estimate the PSD for each of these epochs separately [21]. This socalled, spectrogram is usually computed by means of the short-time Fourier transform (SFFT). However, AR-based spectral estimators can equally be used.

Let $P_{m}^{L}(f)$ denote the estimated PSD of an epoch of the signal $x(n)$ which starts at the $m$-th sample and has a length of $L$ samples. We can then define the AR-based spectrogram as

$$
S(f, n)=P_{n}^{L}(f) .
$$

The better distinction of the peaks that can be obtained through AR estimators means that smaller epoch lengths can be chosen. This allows an increase in time resolution while at the same time maintaining a similar resolution in the frequency domain.

\section{ECG and BCG Signal Analysis}

Both the BCG signals and the lead II ECG signals recorded during our study were first low-pass filtered to $15 \mathrm{~Hz}$ and then downsampled to $30 \mathrm{~Hz}$. Then the signals were split into $5 \mathrm{~s}$ long epochs with $4 \mathrm{~s}$ of overlap, thus resulting in one epoch every second. For each epoch, the PSD was estimated using an AR model of order 50. Spectrograms were then obtained for each signal from the sets of estimated PSDs.

\section{Results and Discussion}

Figure 3 shows the BCG and ECG spectrograms of a healthy reference subject. Both spectrograms show a very similar image containing clear bright lines related to the heart frequency and its harmonics. From a purely visual standpoint, one could conclude that both signals, i.e. the non-contact bed measurement and the reference ECG, contain similar information about the current heart rate of the subject.

Much to our surprise, the spectrograms also showed apparent visual similarities during pathologic episodes of atrial fibrillation. Figure 4 shows the time-frequency analysis of the signals recorded during the treatment session with one of the patients in our study. Before the cardioversion was performed, the
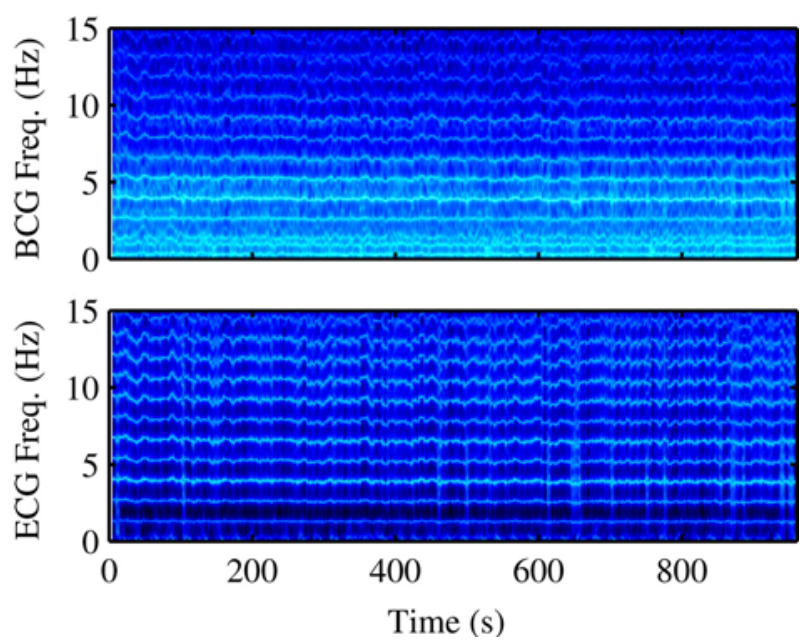

Fig. 3: Spectrograms of simultaneously recorded BCG and ECG signals of a healthy subject, respectively. Both images show clearly visible lines corresponding to the heart rate of the subject and its harmonics. The higher power densities in the lower frequencies of the BCG spectrogram are related to respiratory-induced motions in the BCG signal 


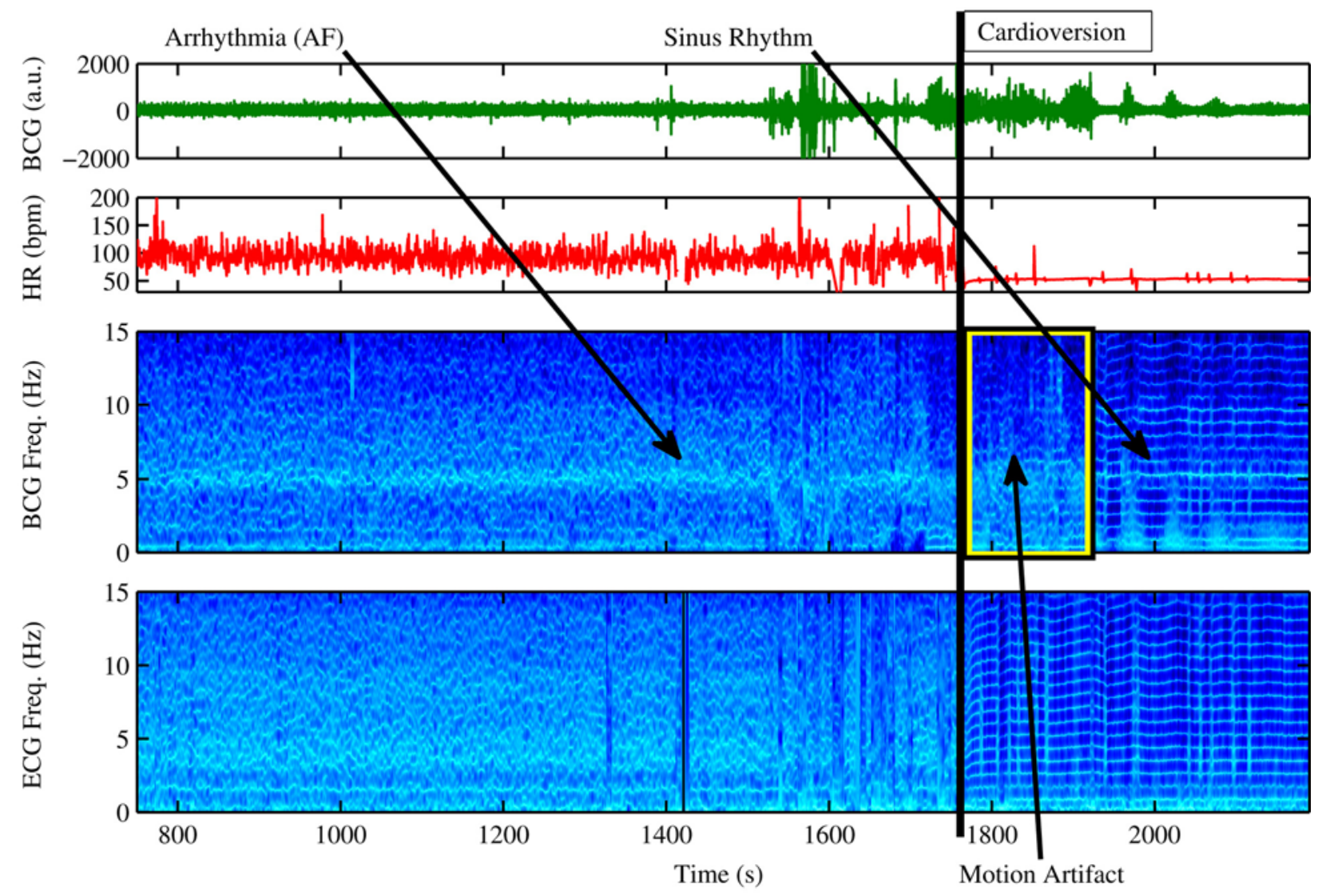

Fig. 4: Time-frequency analysis of the BCG and the reference ECG of patient 11 before and after the cardioversion is performed. Following the cardioversion, both spectrograms change from a noise-like appearance into clearly visible lines representing a base frequency and its harmonics. (From top to bottom: 1. BCG signal recorded by the bed-sensor, 2. beat-to-beat heart rates computed from the reference ECG, 3. spectrogram of the BCG signal, and 4. spectrogram of the reference ECG signal)

patient suffered from atrial fibrillation, which caused strong and rapid fluctuations in the ECG-derived heart rates, as shown in the second plot of the figure. After the cardioversion event, the patient's heart returned to a sinus rhythm and the heart rate stabilized.

When inspecting the spectrograms of the ECG signal and the BCG signal, respectively, the change of state induced by the cardioversion is also immediately visible. While the spectrogram during atrial fibrillation has a smeared, almost noise-like appearance, the spectograms change into the previously seen pattern of distinct lines when the subject's cardiac activity returns to a sinus rhythm. This pattern conforms with what we observed earlier for healthy subjects. In addition to the example shown here, we observed the same differences in spectrogram patterns between arrhythmia and normal periods for all patients that took part in our study.

These preliminary results seem to support our initial hypothesis that arrhythmic cardiac activity can indeed be detected using a bed-mounted BCG system. Furthermore, the observation that ECG and BCG spectrograms undergo the same qualitative changes leads us to believe that the BCG signal recorded using our unobtrusive, non-contact sensor system does indeed contain similar information as the ECG with respect to the presence of arrhythmias.

Nevertheless, our experiments also highlight a major challenge on to road towards a fully automatic BCG-based arrhythmia detector. As shown in Figure 4, motion artefacts immediately after the cardioversion (as evident through the increased amplitude of the BCG signal) cause distortions in the BCG spectrogram which, at first glance, appear similar to arrhythmias. However, an automatic algorithm might still be able to distinguish motion artefacts from arrhythmia by taking the BCG signal amplitude and the details of the frequency distribution into account.

\section{Conclusion}

We have introduced a bed-based sensor system that can unobtrusively monitor the cardiopulmonary activity of a person lying in bed. Unlike previous work 
in the field, which has mostly treated arrhythmias in the data sets as undesirable artefacts, we have devised and presented a clinical study dedicated explicitly to the goal of evaluating the fitness of the proposed system for detecting cardiac arrhythmias. Through the analysis of the data acquired during this study by means of an AR-model-based timefrequency representation, we have shown that the proposed system does indeed enable cardiac arrhythmias to be detected.

Our work prepares the way for future research on fully automatic algorithms for detecting arrhythmias in BCG signals. While the types of arrhythmias which can be detected in BCG recordings is still limited to atrial fibrillation, our findings might facilitate improvements in the long-term management and treatment of cardiac diseases for which AF episodes can be an important marker.

\section{Acknowledgement}

The research presented in this paper was supervised by Prof. S. Leonhardt, RWTH Aachen University in Aachen, Germany, and was sponsored by Philips Research, Eindhoven, the Netherlands.

The author also thanks S. de Waele of Philips Research for insightful discussions. Further thanks go to Prof. P. Schauerte and M. Zink of the Department of Cardiology, Medical Clinic I, University Hospital Aachen, for enabling and supporting the execution of this study.

\section{References}

[1] Organization, W. H.: The World Health Report 2004. 2004. http://www.who.int/whr/2004/ annex/topic/en/annex_2_en.pdf

[2] Gordon, J. W.: Certain Molar Movements of the Human Body produced by the Circulation of the Blood, Journal of Anatomy and Physiology, 1877, vol. 11, p. 533-536.

[3] Inan, O. T., Etemadi, M., Wiard, R. M., Giovangrandi, L., Kovacs, G. T. A.: Robust Ballistocardiogram Acquisition for Home Monitoring, Physiological Measurement, 2009, vol. 30, pp. 169-185.

[4] Brüser, C., Stadlthanner, K., Brauers, A., Leonhardt, S.: Applying Machine Learning to Detect Individual Heart Beats in Ballistocardiograms, In Proc. 32nd Ann. Int. Conf. of the IEEE EMBS, Buenos Aires, Argentina, 2010, pp. 1926-1929.

[5] Kortelainen, J. M., Virkkala, J.: FFT averaging of multichannel BCG signals from bed mattress sensor to improve estimation of heart beat interval, Proc. 29th Ann. Int. Conf. of the IEEE EMBS, Cité Internationale, Lyon, France, 200\%, p. $6685-6688$.

[6] Aubert, X. L., Brauers, A.: Estimation of Vital Signs in Bed from a Single Unobtrusive Mechanical Sensor: Algorithms and Real-life Evaluation, Proc. 30th Ann. Int. Conf. of the IEEE EMBS, British Columbia, Canada, 2008, pp. $4744-4747$.

[7] Chuo, Y., Tavakolian, K., Kaminska, B.: Evaluation of a Novel Integrated Sensor System for Synchronous Measurement of Cardiac Vibrations and Cardiac Potentials, Journal of medical systems, 2009, pp. 1-11.

[8] Zhu, X., Chen, W., Nemoto, T., Kanemitsu, Y., Kitamura, K., Yamakoshi, K., Wei, D.: RealTime Monitoring of Respiration Rhythm and Pulse Rate During Sleep, IEEE Trans. Biomed. Eng., no. 12, vol. 53, 2006, pp. 2553-2563.

[9] Watanabe, K., Watanabe, T., Watanabe, H., Ando, H., Ishikawa, T., Kobayashi, K.: Noninvasive Measurement of Heartbeat, Respiration, Snoring and Body Movements of a Subject in Bed via a Pneumatic Method, IEEE Trans. Biomed. Eng., no. 12, vol. 52, 2005, pp. $2100-2107$.

[10] Chee, Y., Han, J., Youn, J., Park, K.: Air mattress sensor system with balancing tube for unconstrained measurement of respiration and heart beat movements, Physiological Measurement, vol. 26, 2005, pp. 413-422.

[11] Spillman Jr., W. B., Mayer, M., Bennett, J., Gong, J., Meissner, K. E., Davis, B., Claus, R. O., Muelenaer Jr., A. A., Xu, X.: A 'smart' bed for non-intrusive monitoring of patient physiological factors, Measurement Science and Technology, vol. 15, 2004, pp. 1614-1620.

[12] Mack, D. C., Patrie, J. T., Suratt, P. M., Felder, R. A., Alwan, M. A.: Development and preliminary validation of heart rate and breathing rate detection using a passive, ballistocardiography-based sleep monitoring system, IEEE Trans. Inf. Technol. Biomed., no. 1, vol. 13 , 2009, p. 111-120.

[13] Junnila, S., Akhbardeh, A., Värri, A.: An Electromechanical Film Sensor Based Wireless Ballistocardiographic Chair: Implementation and Performance, Journal of Signal Processing Systems, no. 3, vol. 57, 2008, pp. 305-320. 
[14] Go, A. S., Hylek, E. M., Phillips, K. A., Chang, Y., Henault, L. E., Selby, J. V., Singer, D. E.: Prevalence of diagnosed atrial fibrillation in adults: national implications for rhythm management and stroke prevention: the AnTicoagulation and Risk Factors in Atrial Fibrillation (ATRIA) Study, JAMA, no. 18, vol. 285, 2001, pp. 2370-2375.

[15] Katz, A. M.: Physiology of the Heart. M. Lippincott Williams \& Wilkins, 2005.

[16] Maisel, W. H., Stevenson, L. W.: Atrial fibrillation in heart failure: epidemiology, pathophysiology, and rationale for therapy, American Journal of Cardiology, no. 6, vol. 91, 2003, pp. 2D-8D.

[17] Paajanen, M., Lekkala, J., Kirjavainen, K.: ElectroMechanical Film (EMFi): a new multipurpose electret material, Sensors and Actuators A: Physical, no. 1-2, vol. 84, 2000, pp. $95-102$.

[18] Shea, J. B., Maisel, W. H.: Cardioversion, Circulation, no. 22, vol. 106, 2002, pp. e176-e178.

[19] Kay, S. M.: Modern Spectral Estimation: Theory and Application. Prentice Hall, 1999.
[20] Broersen, P.: Finite-sample bias propagation in autoregressive estimation with the YuleWalker method, IEEE Trans. Instrumentation and Measurement, no. 5, vol. 58, 2009, pp. 1354 1360 .

[21] Mitra, S. K.: Digital Signal Processing: A Computer-Based Approach. McGraw-Hill, 2001.

\section{About the author}

Christoph BRÜSER was born in Troisdorf, Germany, in 1983. He holds a Dipl.-Ing. degree in Computer Engineering from RWTH Aachen University, Aachen, Germany. Currently, he is pursuing a Dr.Ing. (Ph.D.) degree in the Department of Medical Information Technology, RWTH Aachen University, where he is also working as a Research Assistant. His research interests include biosignal processing and classification as well as unobtrusive physiological measurement techniques.

Christoph Brüser

E-mail: brueser@hia.rwth-aachen.de

Philips Chair for Medical Information Technology

RWTH Aachen University

Pauwelsstrasse 20, 52074 Aachen, Germany 